\title{
Enhancement in the ${ }^{6} \mathrm{He}^{64} \mathrm{Zn}$ fusion cross section at energies around the barrier: static or dynamic effect?
}

\author{
M. Fisichella ${ }^{1,2}$, V. Scuderi ${ }^{2,3}$, A. Di Pietro ${ }^{2}$, P. Figuera ${ }^{2}$, M. Lattuada ${ }^{2,3}$, C. Marchetta ${ }^{2}$, M. Milin ${ }^{4}$, A. Musumarra ${ }^{2,3}$, \\ M.G. Pellegriti ${ }^{2,3}$, N. Skukan ${ }^{5}$, E. Strano ${ }^{2,3}$, D. Torresi ${ }^{2,3}$, M. Zadro ${ }^{5}$ \\ ${ }^{1}$ Dipartimento di Fisica, Università di Messina, Messina, Italy \\ ${ }^{2}$ INFN- Laboratori Nazionali del Sud and sezione di Catania, Catania, Italy \\ ${ }^{3}$ Dipartimento di Fisica ed Astronomia, Università di Catania, Catania, Italy \\ ${ }^{4}$ Department of Physics Faculty of Science University of Zagreb, Zagreb, Croatia \\ ${ }^{5}$ Ruđer Boŝković Institute, Zagreb, Croatia
}

\begin{abstract}
A new measurement of fusion cross-section for the system ${ }^{4} \mathrm{He}+{ }^{64} \mathrm{Zn}$ was performed at sub-barrier energy in order to cover the same energy region of previous measurements of ${ }^{6} \mathrm{He}+{ }^{64} \mathrm{Zn}$. The fusion crosssection was obtained using an activation technique. From the comparison of the two excitation functions an enhancement of the fusion cross section was observed, at energy below the Coulomb barrier, in the reaction induced by ${ }^{6} \mathrm{He}$ in respect to the one induced by ${ }^{4} \mathrm{He}$.
\end{abstract}

\section{Introduction}

In recent years the fusion reaction mechanism in collision induced by halo nuclei has been subject of intense theoretical and experimental studies. It is expected that the structure of these nuclei may affect the reaction mechanism. Direct processes seem to be favoured due to the low binding energy of valence nucleons. Concerning the fusion mechanism an enhancement of the fusion cross section at energies around and below the Coulomb barrier is expected, due to the presence of the diffuse halo which affects the shape of projectile-target potential reducing the Coulomb barrier. Besides of these static effects it is necessary to consider the dynamic effects which are due to the coupling of the relative motion of projectile and target to their intrinsic excitations or to the other reactions channels. In particular coupling with the breakup may be important. Despite of a lot of theoretical and experimental studies that have been done, the role of these dynamic effects it is not still clear. According to the Continuum-Discretised Coupled-Channel (CDCC) calculations [1,2] these dynamic effects should cause an enhancement of the sub-barrier total fusion cross-section with respect to the nocoupling case; on the other hand the high probability of the break-up process should generate a suppression of the total fusion cross-section at energies above the barrier. Opposite results have been obtained using a different approach [3] based on a time dependent wave-packet formalism which uses a three body model (core, halo and target). According to this model, the cross-section in the neutron halo case is slightly suppressed compared to the non-halo case.

Also from the experimental point of view different authors did not reach similar conclusions about the enhancement/suppression effect on the fusion cross section. Fusion reactions induced by the $2 \mathrm{n}$ halo ${ }^{6} \mathrm{He}$ nucleus in the energy region around the Coulomb barrier are the most studied of any involving light radioactive beams. Usually the fusion cross section of the reactions induced by ${ }^{6} \mathrm{He}$ are compared with those obtained using ${ }^{4} \mathrm{He}$ beam on the same target. The ${ }^{4} \mathrm{He}$ represents the ${ }^{6} \mathrm{He}$ core and by comparing the two systems one can observe the effect due to the presence of valence neutrons. The following system have been studied: ${ }^{6} \mathrm{He}+{ }^{209} \mathrm{Bi}$ [4-7], ${ }^{6} \mathrm{He}+{ }^{238} \mathrm{U}$ [8-9], ${ }^{6} \mathrm{He}+{ }^{208} \mathrm{~Pb}[10],{ }^{6} \mathrm{He}+{ }^{63,65} \mathrm{Cu}$ [11] and ${ }^{11} \mathrm{Be}+{ }^{209} \mathrm{Bi}[12-14]$.

Concerning the ${ }^{6} \mathrm{He}+{ }^{209} \mathrm{Bi}$ fusion reaction, the authors found an enhancement of the fusion cross section below the barrier and a strong contribution due to the transfer and/or breakup reaction channels. Also for the ${ }^{6} \mathrm{He}+{ }^{238} \mathrm{U}$ a large enhancement of fission around and below the barrier in the ${ }^{6} \mathrm{He}$ case with respect to the ${ }^{4} \mathrm{He}$ ones was observed. The analysis allowed to conclude that the observed fission enhancement was mostly due to transferfission events and almost no effects on fusion due to the structure of ${ }^{6} \mathrm{He}$ are observed.

For the ${ }^{6} \mathrm{He}+{ }^{206} \mathrm{~Pb}$ reaction, the $2 \mathrm{n}$ evaporation channel was compared to the measured $1 \mathrm{n}$ evaporation channel of ${ }^{4} \mathrm{He}+{ }^{208} \mathrm{~Pb}$. An enhancement of the sub-barrier fusion cross-section for the ${ }^{6} \mathrm{He}$ induced reaction is observed with respect to the ${ }^{4} \mathrm{He}$ ones. According to a model of 
"sequential fusion" proposed by Zagrebaev [15] the enhancement should be attributed to the coupling to the n-transfer channel. Despite the two reactions, ${ }^{6} \mathrm{He}+{ }^{206} \mathrm{~Pb}$ and ${ }^{4} \mathrm{He}+{ }^{208} \mathrm{~Pb}$, form the same compound nucleus $\left({ }^{212} \mathrm{Po}\right)$, it is formed at different excitation energies and in the reaction with ${ }^{4} \mathrm{He}$ beam, it is below the particle threshold. Therefore it would be necessary to compare the measured total fusion cross-section for the two reactions ${ }^{4,6} \mathrm{He}+$ ${ }^{208} \mathrm{~Pb}$ rather than the excitation function for one particular evaporation channel.

In the case of ${ }^{6} \mathrm{He}+{ }^{63,65} \mathrm{Cu}$ fusion reaction, it was observed ${ }^{66} \mathrm{Cu}$ residue cross-sections much larger than the ones calculated using the statistical model. This result suggested that the dominant production mechanism was other than fusion-evaporation, most probably neutron transfer.

The only low energy fusion reaction studies involving the 1n halo ${ }^{11} \mathrm{Be}$ concerns the system ${ }^{11} \mathrm{Be}+{ }^{209} \mathrm{Bi}$. The crosssection data, for the weakly bound one-neutron halo ${ }^{11} \mathrm{Be}$, were compared with those measured for the well bound ${ }^{10} \mathrm{Be}$ nucleus and the weakly bound ${ }^{9} \mathrm{Be}$. The complete fusion cross-section reported in [14] for the three reactions ${ }^{9,10,11} \mathrm{Be}+{ }^{209} \mathrm{Bi}$ show no difference within the errors.

\section{${ }^{4,6} \mathrm{He}+{ }^{64} \mathrm{Zn}$ experiment}

\subsection{Activation technique}

We had performed $[16,17]$ the ${ }^{6} \mathrm{He}+{ }^{64} \mathrm{Zn}$ reaction at the Centre of Research of Cyclotron in Louvain la Neuve to measure the fusion excitation function at energies below and around the Coulomb barrier. The data were compared with those of the reaction ${ }^{4} \mathrm{He}+{ }^{64} \mathrm{Zn}$ measured in the same experiment. The experimental set-up is described in $[16,17]$. To measure the fusion cross-section we used an activation technique based on the off-line measurement of the atomic X-ray emission following the electron capture (E.C.) decay of the evaporation residues (E.R.) produced in the reaction. Direct E.R. detection is very difficult because a large fraction of them will not come out from the target since their kinetic energy is too small. However by choosing, with the help of statistical model calculation, a suitable target, as in our case, it is possible to obtain E.R. unstable against E.C. decay and so it is possible "to detect" the E.R. by looking at X-ray emitted in their decay.

To limit the beam time request, a stack of thick ${ }^{64} \mathrm{Zn}$ targets, each followed by ${ }^{93} \mathrm{Nb}$ catcher, was irradiated. The catchers were needed in order to stop the small fraction of E.R. emerging from the previous target and to slow down the beam, increasing the average difference in beam energy for the different targets. In this way it is possible to extract the cross section at different energies without changing the beam energy.

The beam current was determined from the elastic scattering at small angles where the elastic cross section is known to be Rutherford.

At the end of the irradiation time the activated target and the corresponding catcher are placed very close to a ORTEC $\mathrm{Si}(\mathrm{Li})$ detector, surrounded by lead shields, to measure the residues activity. Possible reactions induced by the beam on the $\mathrm{Nb}$ catchers do not represent a problem since the X-ray energies are different than the ones corresponding to reactions on ${ }^{64} \mathrm{Zn}$. Each measurement was repeated in order to measure the activity as a function of time. The fusion cross-section could be obtained by summing up the cross-sections of all evaporation channels. We had performed a comparison of the measured cross-section for the different channels with a statistical model calculation, performed with the CASCADE code to investigate if there were some contributions from other reaction processes. This comparison showed a good agreement except for the ${ }^{65} \mathrm{Zn}$ residue, were a large enhancement with respect to the calculation was observed. The excess in the yield measured for this channel was attributed to one and two neutron transfer reactions. Therefore, we have subtracted the contribution due to the transfer by replacing the measured value for ${ }^{65} \mathrm{Zn}$ with the one calculated using the statistical model calculations. No evident effects were observed for ${ }^{6} \mathrm{He}+{ }^{64} \mathrm{Zn}$ fusion with respect to ${ }^{4} \mathrm{He}+{ }^{64} \mathrm{Zn}$ within the measured energy range but we cannot say anything about the role of halo structure at energies below the Coulomb barrier because the ${ }^{6} \mathrm{He}$ data extended to lower energies than the ${ }^{4} \mathrm{He}$ ones.

\section{New ${ }^{4} \mathrm{He}+{ }^{64} \mathrm{Zn}$ experiment}

To cover the lower energy region of the ${ }^{6} \mathrm{He}$ data a new ${ }^{4} \mathrm{He}+{ }^{64} \mathrm{Zn}$ experiment was performed at Ruder Boskovic Institute in Zagreb. The fusion excitation function was measured in an energy range from 7 to $9 \mathrm{MeV}$ and as in our previous experiment, it was measured by using the activation technique. This time the ${ }^{64} \mathrm{Zn}\left(\approx 540 \mu \mathrm{g} / \mathrm{cm}^{2}\right)$ targets were irradiated one by one together with the corresponding ${ }^{93} \mathrm{Nb}\left(\approx 1000 \mu \mathrm{g} / \mathrm{cm}^{2}\right)$ catcher and the beam current was measured using a faraday cup. The irradiated target was measured at Laboratori Nazionali del Sud for about twenty days.

Typical X-ray spectrum measured off-line for the reaction ${ }^{4} \mathrm{He}+{ }^{64} \mathrm{Zn}$ is shown in the inset of figure 1 . The two peaks correspond to the $\mathrm{K}_{\alpha}$ and $\mathrm{K}_{\beta} \mathrm{X}$-ray emission of $\mathrm{Zn}$. In the present experiment, the analysis was performed taking into consideration only the $K_{\alpha}$ lines.

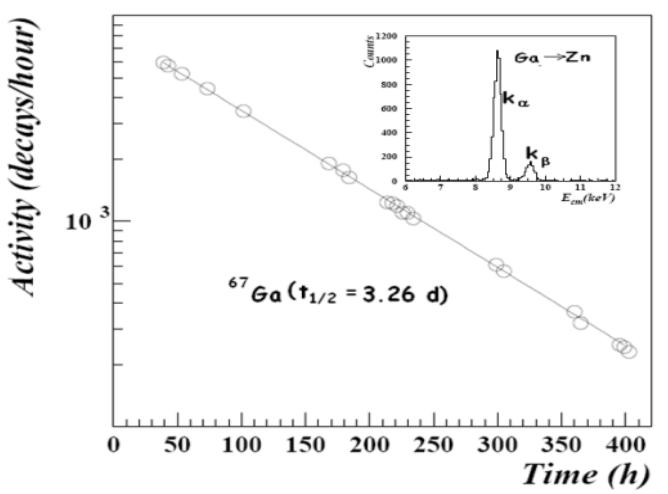

Fig.1 Activity curve for the ${ }^{67} \mathrm{Ga}$ isotope extracted in the activation run. In the inset a typical X-ray spectrum measured off-line for the reaction ${ }^{4} \mathrm{He}+{ }^{64} \mathrm{Zn}$ is shown. It is possible to distinguish two peaks which correspond to the $\mathrm{K}_{\alpha}$ and $\mathrm{K}_{\beta}$ 
The E.R. can be identified by atomic number from the energy of the X-ray lines, whereas it is possible to discriminate the different isotope contributions by their half- lives, following the X-ray activity as a function of time. In figure 1 the activity curve for the $\mathrm{Ga}$ isotope is shown.

As one can see from the slope of the activity curve only the contribution of ${ }^{67} \mathrm{Ga}$ isotope is present. By using statistical model calculation we had estimated that not only ${ }^{67} \mathrm{Ga}$ but also and ${ }^{67} \mathrm{Ge}$ should be produced in this reaction. We detected only the first one since ${ }^{67} \mathrm{Ge}$ is a shortlived nucleus $\left(\mathrm{t}_{1 / 2}=18\right.$ minutes $)$ and it decays by E.C. $100 \%$ into ${ }^{67} \mathrm{Ga}$. Therefore, after one day, time at which the off-line measurement started, we observed only the ${ }^{67} \mathrm{Ga}$ contribution which, however, is the sum of both ${ }^{67} \mathrm{Ge}$ and ${ }^{67} \mathrm{Ga}$.

The total fusion cross section corresponds to the longer lived ${ }^{67} \mathrm{Ga}\left(\mathrm{T}_{1 / 2}=3.26 \mathrm{~d}\right)$ production cross section. In figure 2 data of ${ }^{4} \mathrm{He}+{ }^{64} \mathrm{Zn}$ (closed stars) are plotted together the previous data of ${ }^{4,6} \mathrm{He}+{ }^{64} \mathrm{Zn} \mathrm{[16].} \mathrm{The}$ consistency of the two data sets is guaranteed by the good agreement of the points around $9.5 \mathrm{MeV}$.

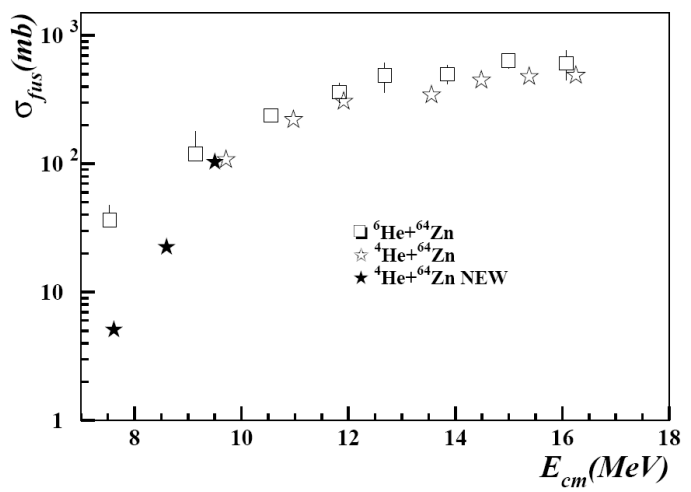

Fig.2 ${ }^{6} \mathrm{He}+{ }^{64} \mathrm{Zn}$ (squares) and ${ }^{4} \mathrm{He}+{ }^{64} \mathrm{Zn}$ (stars) fusion excitation function. The ${ }^{6} \mathrm{He}$ and ${ }^{4} \mathrm{He}$ at upper energies (open stars) are the data of the previous experiment $[16,17]$. With the closed stars are plotted the new preliminary ${ }^{4} \mathrm{He}$ data

An enhancement of the fusion excitation functions at energies below the Coulomb barrier in the case of ${ }^{6} \mathrm{He}$ with respect the ${ }^{4} \mathrm{He}$ one is observed. The enhancement is attributed to the halo structure of ${ }^{6} \mathrm{He}$, but from this it is not possible to disentangle between static and dynamic effects. In order to discriminate the static contribution it is necessary to reduced the data by normalizing the fusion cross section by the geometrical factor $\left(R_{B}\right)$ and subtracting from the energy the height of the Coulomb barrier $\mathrm{V}_{\mathrm{B}}$. This normalization procedure has been performed by using radius and barrier height values taken from [18] and obtained by fitting the target projectile potential obtained with a double folding procedure using realistic densities. The preliminary results of this procedure are shown in figure 3.

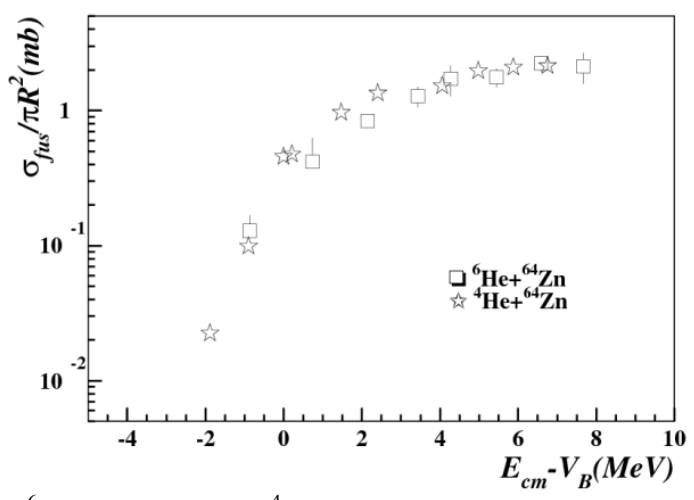

Fig. $3{ }^{6} \mathrm{He}$ (squares) and ${ }^{4} \mathrm{He}$ (stars) preliminary data reduced for the respective radius and barrier height. These values have been taken from [18].

After the normalization, the difference between the two excitation functions disappears thus suggesting that only static effects contribute to the enhancement of the ${ }^{6} \mathrm{He}$ induced cross-section.

\section{Conclusion}

Despite of a lot experiments have been performed to study the fusion mechanisms in collisions induced by halo nuclei, a clear systematic behavior for all systems is not yet observed. In general it is important to notice that most of the existing data do not really explore the region below the barrier with reasonable errors and it is not always clearly discussed which is the role played by static and dynamic effects in the fusion mechanism. Concerning our measurement it has been observed an enhancement of the ${ }^{6} \mathrm{He}+{ }^{64} \mathrm{Zn}$ fusion cross section with respect to ${ }^{4} \mathrm{He}+{ }^{64} \mathrm{Zn}$ at energies around and below the Coulomb barrier. This behavior is surely linked to the halo structure of the ${ }^{6} \mathrm{He}$ and it seems to be correlated to static effects.

\section{References}

1. K. Hagino et al., Phys. Rev. C 61, 037602 (2002)

2. A. Diaz-Torres, J.J. Thompson, Phys. Rev.C 65, 24606 (2002)

3. M. Ito, et al., Phys. Lett. B 37, 53 (2006)

4. P. A. De Young et al., Phys. Rev. C 58, 3442 (1998)

5. J. J. Kolata et al., Phys. Rev. Lett. 81, 4580 (1998)

6. E. F. Aguilera et al., Phys. Rev. Lett. 84, 5058 (2000)

7. E. F. Aguilera et al., Phys. Rev. C 63, 061603(R) (2001)

8. M. Trotta et al., Phys. Rev. Lett. 84, 2342 (2000)

9. R. Raabe et al. Nature 431, 823(2004)

10. Y.E. Penionzhkevich et al. Phys. Rev. Lett. 96, 162701 (2006)

11. A. Navin et al., Phys. Rev. C 70, 044601 (2004)

12. C. Signorini et al., Eur. Phys. J. A 2, 227 (1998)

13. C. Signorini et al. Eur. Phys. J. A 5, 7 (1999)

14. C. Signorini et al. Nucl. Phys. A 735, 329 (2004)

15. V.I. Zagrebaev Phys. Rev. C 67, 061601(R) (2003) 
EPJ Web of Conferences

16. A. Di Pietro et al. Phys. Rev. C 69, 044613 (2004)

17. A. Di Pietro et al. Eur.Phys.J. Special Topics 150, 15 (2007)

18. L.F.Canto et al., Nucl. Phys. A 821, 51 (2009) 